\title{
SEMI-SYMMETRIC SUBMANIFOLDS WITH MAXIMAL FIRST NORMAL SPACE
}

\author{
U. LUMISTE. POOLSUMMEETRILISED ALAMMUUTKONNAD MAKSIMAALSE ESIMESE NOR- \\ MAALRUUMIGA
}

Ю. ЛУМИСТЕ. ПОЛУСИММЕТРИЧЕСКИЕ ПОДМНОГООБРАЗИЯ С МАКСИМАЛЬНЫМ ПЕР. ВЫМ НОРМАЛЬНЫМ ПРОСТРАНСТВОМ

\section{(Presented by H. Keres)}

1. A Riemannian manifold $M^{m}$ satisfying $R(X, Y) \cdot R=0$ (i.e. the integrability condition of the system $\nabla R=0$ which characterizes a symmetric Riemannian manifold) is called a semi-symmetric Riemannian manifold $\left[{ }^{1,2}\right]$. Analogically, a submanifold $M^{m}$ in a Euclidean $E^{n}$ satisfying $\bar{R}(X, Y) \cdot h=0$ (i.e. the integrability condition of the system $\bar{\nabla} h=0$ which characterizes a symmetric $M^{m}$ in $E^{n}\left[{ }^{3}\right]$ ), is called a semi-symmetric submanifold $M^{m}$ in $E^{n}$; cf. [ ${ }^{4}$ ], where, the term «semi-parallel» is used. Intrinsically it is a semi-symmetric Riemannian manifold. Here $h$ is the second fundamental form and $\bar{\nabla}$ is the van der Waerden-Bortolotti connection [ $\left.{ }^{5}\right]$.

A problem is known in the theory of semi-symmetric Riemannian manifolds, $\left[{ }^{6}\right]$ : are the only semi-symmetric irreducible manifolds the symmetric ones? The answer is, in general, negative $\left[{ }^{7}\right]$, but can be positive through some additional conditions.

A similar problem rises in the theory of semi-symmetric submanifolds $M^{m}$ in $E^{n}$ in what conditions the only semi-symmetric submanifolds are symmetric. We are trying to give an answer to this problem in the following.

Note that all semi-symmetric surfaces $(m=2)$ and hypersurfaces $(m=n-1)$ in $E^{n}$ are classified in $\left.{ }^{[}\right]$and $\left[{ }^{8}\right]$, respectively. All semisymmetric $M^{n-2}$ in $E^{n}$ are described in [ $\left.{ }^{9}\right]$. Most of them are not symmetric.

To formulate our main result, we recall that the linear span of all $h(X, X)$ in a given point $x \in M^{m}$ for arbitrary $X \in T_{x} M^{m}$ is called the first normal space of the submanifold $M^{m}$, and is denoted by $N_{x} M^{m}$, The Euclidean subspace, spanned on $x \in M^{m}, T_{x} M^{m}$ and $N_{x} M^{m}$, is called the first osculating space of the submanifold $M^{m}$ in the point $x$.

Theorem. Every semi-symmetric submanifold $M^{m}$ in $E^{1 / 2 m(m+3)}$, $m \geqslant 2$, for which $E^{1 / 2 m(m+3)}$ is the first osculating space in every point $x \in M^{m}$, is a symmetric one, it has the inner metric of positive constant curvature and, in the case of its completeness, coincides with an orbit of the orthogonal group $O(m+1)$ acting in $E^{1 / 2 m(m+3)}$ by isometries.

Note that in case $m=2$, this orbit is the classical Veronese surface $\left[{ }^{10}\right]$ in a 4 -sphere $S^{4} \subset E^{5}$ (see also [5], p. 88). In this case our theorem complements the classification theorem in $\left.{ }^{4}\right]$. If $m>2$, this orbit is an 
elliptic $m$-space minimally embedded in $S^{\frac{1}{2} m(m+3)-1}$ $m$-submanifold»; see $\left.\left[{ }^{11-13}\right]\right)$.

We obtain the Theorem as a consequence from a more general result (see Preposition below).

2. Let $\left\{x ; e_{1}, \ldots, e_{m} ; e_{m+1}, \ldots, e_{n}\right\}$ be the moving orthogonal frame adapted to the submanifold $M^{m}$ in $E^{n}$, i. e. let $x \in M^{m}$ and $e_{i} \in T_{x} M^{m}$, $e_{\alpha} \in T \perp{ }_{x} M^{m} ; i, j, \ldots=1, \ldots, m ; \alpha, \beta, \ldots=m+1, \ldots, n$. If we identify the point $x$ with its radius vector in $E^{n}$, then in the derivation formulae $d x=e_{I} \omega^{I}, d e_{I}=e_{J} \omega_{I}^{J}, \omega_{I}^{J}+\omega_{J}^{I}=0 ; I, J=1, \ldots, n$; we have

$$
\begin{gathered}
\omega^{\alpha}=0, \\
\omega_{i}^{\alpha}=h_{i j}^{\alpha} \omega^{j}, \quad h_{i j}^{\alpha}=h_{j i}^{\alpha}, \\
\bar{\nabla} h_{i j}^{\alpha}:=d h_{i j}^{\alpha}-h_{k j}^{\alpha} \omega_{i}^{k}-h_{i k}^{\alpha} \omega_{j}^{k}+h_{i j}^{\beta} \omega_{\beta}^{\alpha}=h_{i j k}^{\alpha} \omega^{k}, \quad h_{i j k}^{\alpha}=h_{i k j}^{\alpha}, \\
\bar{\nabla} h_{i j k}^{\alpha} \wedge \omega^{k}=-h_{k j}^{\alpha} \Omega_{i}^{k}-h_{i k}^{\alpha} \Omega_{j}^{k}+h_{i j}^{\beta} \Omega_{\beta}^{\alpha} .
\end{gathered}
$$

Here

$$
\begin{aligned}
& \Omega_{i}^{j}:=d \omega_{i}^{j}-\omega_{i}^{h} \wedge \omega_{k}^{j}=-\sum_{\alpha} h_{i[k}^{\alpha} h_{l] j}^{\alpha} \omega^{k} \wedge \omega^{l}, \\
& \Omega_{\alpha}^{\beta}:=d \omega_{\alpha}^{\beta}-\omega_{\alpha}^{\gamma} \wedge \omega_{\gamma}^{\beta}=-\sum_{i} h_{i[k}^{\alpha} h_{l] i}^{\beta} \omega^{k} \wedge \omega^{l}
\end{aligned}
$$

are the curvature 2 -forms of the Levi-Civita connection $\nabla$ and of the normal connection $\nabla^{\perp}$, respectively; they all represent the curvature 2 -forms of the van der Waerden-Bortolotti connection $\bar{\nabla}=\nabla \oplus \nabla^{\perp}$. Their last expressions follow from the structure equations $d \omega^{I}=\omega^{J} \wedge \omega_{J}^{I}$, $d \omega_{I}^{J}=\omega_{I}^{K} \wedge \omega_{K}^{J}$ due to (2). Each of the formulae (2)-(4) can be obtained from the previous one after exterior differentiation and by using the Cartan lemma, if needed.

Denoting $h(X, Y)=h^{\alpha}{ }_{i j} X^{i} Y^{j} e_{\alpha}$ for $X=e_{i} X^{i}$ and $Y=e_{j} Y^{j}$, we have the second fundamental form $h$. Then $h_{i j k}^{\alpha}=\bar{\nabla}_{k} h_{i j}^{\alpha}$ are the components of $\bar{\nabla} h$ and the last equality (3) is the Peterson-Codazzi equation.

3. A submanifold $M^{m}$ in $E^{n}$ is said to be symmetric [ [ ${ }^{3}$ if its second fundamental form $h$ is parallel, i. e. if $\bar{\nabla} h=0$ or if $h_{i j k}^{\alpha}=0$ in (3). Intrinsically it is a locally symmetric space, more exactly, a symmetric $R$-space, and its immersion in $E^{n}$ is a standard one $\left[{ }^{3}\right]$. From (4) it follows that in the case of symmetricity we have

$$
h_{k j}^{\alpha} \Omega_{i}^{k}+h_{i k}^{\alpha} \Omega_{j}^{k}-h_{i j}^{\beta} \Omega_{\beta}^{\alpha}=0 .
$$

This is the component form of $\bar{R}(X, Y) h=0$ and is, as we see, a system of cubic equations on the components of $h$. In general, a submanifold $M^{m}$ in $E^{n}$, satisfying (7), is said to be semi-symmetric.

4. Let $m_{1}=\operatorname{dim} N_{x} M^{m}$ be constant on $M^{m}$; of course $m_{1} \leqslant \frac{1}{2} m(m+1)$. If $n>m+m_{1}$, the moving orthonormal frame $\left\{x ; e_{m+1}, \ldots, e_{m+m_{1}}\right.$; $\left.e_{m+m_{1}+1}, \ldots, e_{n}\right\}$ can be adapted so that $e_{\rho} \in N_{x} M^{m}, \quad e_{\xi} \in N_{x}^{\perp} M^{m}$; $\varrho, \sigma, \ldots=m+1, \ldots m+m_{1} ; \xi, \eta, \ldots=m+m_{1}+1, \ldots, n$. Then $h_{i j}^{\xi}=0$ and the system of relations $h_{i j}=h_{i j}^{\rho} e_{\rho}$ is invertible. From (3) it follows that $h_{i j}^{\rho} \omega_{\rho}^{\xi}=h_{i j k}^{\xi} \omega^{k}$, and this gives $\omega_{\rho}^{\xi}=\chi_{\rho k}^{\xi} \omega^{k}$. Now 


$$
\tilde{\Omega}_{\rho}^{\sigma}:=d \omega_{\rho}^{\sigma}-\omega_{\rho}^{\tau} \wedge \omega_{\tau}^{\sigma}=-\left(\sum_{i} h_{i[k}^{\rho} h_{l] i}^{\sigma}+\sum_{\xi}^{\beta} \chi_{\rho[k}^{\xi} \chi_{[\sigma] l]}^{\xi}\right) \omega^{k} \wedge \omega^{l} .
$$

Denoting $N M^{m}=\bigcup_{x \in M^{m}} N_{x} M^{m}$, we have the first normal vector bundle $N M^{m} \rightarrow M^{m}$ with fibre $N_{x} M^{m}$ on every $x \in M^{m}$. The fact that 2 -forms $\tilde{\Omega}_{\rho}^{\sigma}$ are, according to $(8)$, semi-basic, shows that there is a connection in this bundle with connection forms $\omega_{\rho}^{\sigma}$ (see $\left[{ }^{14}\right]$, Ch. II, $\S 1$ ). This connection will be denoted $\nabla^{N}$ and called the first normal connection. In the first osculating bundle $\bigcup_{x \in M^{m}} T_{x} M^{m} \oplus N_{x} M^{m} \rightarrow M^{m}$ we have the connection $\bar{\nabla}^{1}=\nabla \oplus \nabla^{N}$. If

$$
\overrightarrow{\nabla^{1}} h_{i j}^{\rho}:=d h_{i j}^{\rho}-h_{k j}^{\rho} \omega_{i}^{k}-h_{i k}^{\rho} \omega_{j}^{k}+h_{i j}^{\sigma} \omega_{\sigma}^{\rho}=0 \text {, }
$$

in short $\bar{\nabla}^{1} h=0$, then $h$ is said to be first-order parallel.

$\mathrm{L}$ e $\mathrm{mm}$. The parallelism of the second fundamental form $h$ (i.e. $\bar{\nabla} h=0)$ yields its first-order parallelism $\left(i . e . \bar{\nabla}^{1} h=0\right)$. The converse is true iff $M^{m}$ lies in its first osculating space, i.e. iff $T_{x} M^{m} \oplus N_{x} M^{m}$ is independent from $x \in M^{m}$.

In fact, the last assertion is equivalent to $\omega_{\rho}^{\xi}=0$, and so is also the converse of the first assertion.

5. Proposition. If a submanifold $M^{m}$ in $E^{n}(m \geqslant 3, n \geqslant$ $\left.\geqslant \frac{1}{2} m(m+3)\right)$ is semi-symmetric and its first normal space $N_{x} M^{m}$ has maximal dimension $\frac{1}{2} m(m+1)$ in every point $x \in M^{m}$, then $M^{m}$ is intrinsically a space of positive constant curvature and, as a submanifold, it has the first-order parallel second fundamental form.

Proof. Denoting $\sum_{\rho} h_{i j}^{\rho} h_{h l}^{\rho}=B_{i j, k l}$, we can write the first assumption, i. e. (7), in the form

$$
\sum_{k}\left(h_{k j} B_{i[p, q] k}+h_{i k} B_{j[p, q] k}-B_{i j, k[p} h_{q] k}\right)=0,
$$

where $h_{i j}=h_{i j}^{\alpha} e_{\alpha}=h_{i j}^{\rho} e_{\rho}$. The second assumption says that these vectors $h_{i j}$ are linearly independent and thus from (9) we have

$$
B_{i j, k l}=x^{2}\left(2 \delta_{i j} \delta_{k l}+\delta_{i k} \delta_{j l}+\delta_{i l} \delta_{j k}\right) .
$$

In fact, it follovs from (9) that for every three distinct values $a, b$ and $c$

$$
B_{a a, a a}=2 B_{a a, b b}=4 B_{a b, a b}=4 \varkappa^{2}, \quad B_{a a, a b}=B_{a a, b c}=B_{a b, a c}=0 ;
$$

if $m \geqslant 4$, then also $B_{a b, c d}=0$ for distinct $a, b, c, d$.

Due to (5) and (10)

$$
\Omega_{i}^{j}=-x^{2} \omega^{i} \wedge \omega^{j} .
$$

The Schur theorem gives now $x=$ const and so the first assertion of the Proposition is proved.

Consequently, $\sum h_{i j}^{\rho} h_{k l}^{\rho}=$ const and after differentiation, using by $h_{i j}^{\xi}=0$, we get $^{\rho}$

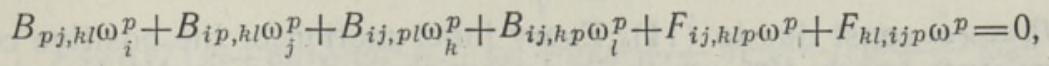

where $F_{i j, k l p}=\sum h_{i j}^{\rho} h_{k l p}^{\rho}$ are symmetric with respect to indices in the 
first pair and also in the second triplet. After substituting here (10), we get $F_{i j, k l p}=-F_{k l, i j p}$ and, using this symmetry, we have $F_{i j, k l p}=$ $=-F_{k l, i p j}=F_{i p, k l j}$, so that every index in the first pair can be exchanged by every index in the second triplet. Thus $F_{i j, k l p}=F_{k l, i j p}$ and this, together with $F_{i j, k l p}=-F_{k l, i j p}$, gives $F_{i j, k l p}=0$. It follows that $h_{k l p}^{\rho}=0$. This is equivalent to the second assertion of the Proposition.

6. Now, the first two assertions of the Theorem in case $m \geqslant 3$ follow from the Proposition and Lemma.

In case $m=2$ when (12) does not give $x=$ const, it can be deduced independently from the assumption of the Theorem. Here (11) shows that we can take an orthonormal frame $\left\{x ; e_{3}, e_{4}, e_{5}\right\}$ in every $T{ }_{x}^{\perp} M^{2}=$ $=N_{x} M^{2}$, so that

$$
h_{11}=x\left(\sqrt{3} e_{3}+e_{4}\right), \quad h_{22}=x\left(\sqrt{3} e_{3}-e_{4}\right), \quad h_{12}=x e_{5} .
$$

Then, in $d e_{i}=e_{j} \omega_{i}^{j}+e_{\alpha} \omega_{i}^{\alpha}$ we have (cf. [ $\left.{ }^{4}\right]$ )

$$
\begin{array}{lll}
\omega_{1}^{3}=x \sqrt{3 \omega^{1}}, & \omega_{1}^{4}=x \omega^{1}, & \omega_{1}^{5}=x \omega^{2}, \\
\omega_{2}^{3}=x \sqrt{3 \omega^{2}}, & \omega_{2}^{4}=-x \omega^{2}, & \omega_{2}^{5}=x \omega^{1} .
\end{array}
$$

The differential prolongation gives that

$$
\begin{aligned}
-\frac{1}{2} d \ln x & =A \omega^{1}+B \omega^{2}, & \frac{1}{5}\left(2 \omega_{1}^{2}-\omega_{4}^{5}\right) & =-B \omega^{1}+A \omega^{2}, \\
\frac{1}{\sqrt{3}} \omega_{3}^{4} & =A \omega^{1}-B \omega^{2}, & \frac{1}{\sqrt{3}} \omega_{3}^{5} & =B \omega^{1}+A \omega^{2}
\end{aligned}
$$

and then

$$
\begin{aligned}
& d A=B \omega_{1}^{2}+\frac{1}{5}\left(14 B^{2}-11 A^{2}\right) \omega^{1}-5 A B \omega^{2}, \\
& d B=-A \omega_{1}^{2}-5 A B \omega^{1}+\frac{1}{5}\left(14 A^{2}-11 B^{2}\right) \omega^{2} .
\end{aligned}
$$

Now the exterior differentiation yields

$$
A\left[x^{2}+\frac{42}{25}\left(A^{2}+B^{2}\right)^{7}=B\left[x^{2}+\frac{42}{25}\left(A^{2}+B^{2}\right)\right]=0,\right.
$$

thus $A=B=0$ and $x=$ const.

Repeating, by assumptions of the Theorem, the last part in the proof of the Proposition we get in case $m=2$, too, the parallelism of $h$, i. e. the symmetricity of $M^{m}$.

7. It remains to prove the last assertion of the Theorem. The submanifold $M^{m}$ considered above lies in the hypersphere $S^{n-1}, n=\frac{1}{2} m(m+3)$, because the point $c$ with the radius vector

$$
c=x+\frac{m}{2 x^{2}(m+1)} H
$$

is fixed, i.e. $d c=0$, where $H=\frac{1}{m} \sum_{i} h_{i i}$ is the mean curvature vector, and $\|H\|=x=$ const due to (11). Every inner motion of the positive constant curvature space $M^{m}$, which is determined infinitesimally by $\omega^{i}$ and $\omega_{i}^{j}$, is a rotation in $E^{n}$ about $c$. In fact, by this infinitesimal displacement 
we have $d x=e_{i} \omega^{i}, d e_{i}=e_{j} \omega_{i}^{j}+h_{i j} \omega^{j}, \omega_{i}^{j}+\omega_{j}^{i}=0, d h_{i j}=-\sum_{k} B_{i j, k l} e_{k} \omega^{i}+$ $+h_{k j} \omega_{i}^{k}+h_{i k} \omega_{j}^{k}$, where (10) holds, and then $\left\langle e_{i}, e_{j}\right\rangle,\left\langle e_{i}, h_{k l}\right\rangle$ and $\left\langle h_{i j}, h_{k l}\right\rangle$ conserve their values, respectively $\delta_{i j}, 0$ and $B_{i j, k l}$, as is easy to see by differentiation.

In the case of completeriess of $M^{m}$ the universal covering group for all these inner motions is $O(m+1)$, which acts on $E^{n}$ by rotations with centre $c$, and $M^{m}$ is its orbit in $S^{n \rightarrow 1} \subset E^{n}$. So the Theorem is proved.

\title{
REFERENCES
}

1. Синюков Н. С. Геодезические отображения римановых пространств. М., Наука, 1979 (Гл. II, § 3).

2. Szabí, Z. I. // J. Differ. Geometry, 1982, 17, 531-582.

3. Ferus, D. // Math. Ann., 1980, 247, 81-93.

4. Deprez, J. // J. of Geometry, 1985, 25, 192-200.

5. Chen, B.-Y. Geometry of Submanifolds. New-York, Marcel Dekker. Inc., 1973.

6. Nomizu, K. // Tõhoku Math. J., 1968, 20, 46-59.

7. Takagi, H. // Tōhoku Math. J., 1972, 24, 105-108.

8. Deprez, J. // Rend. Semin. mat. Univ. e politecn. Torino, 1987, 44, 303-316.

9. Lumiste, U. // Acta et comm. Univ. Tartuensis, 1988, 803, 79-94.

10. Borüvka, O. // C. r. Acad. sci., 1928, 187, 334-336.

11. Blanuša, D. // Glas. mat.-fiz. i astron., 1953, 8, 81-114.

12. Солодовников А. С. // Тр. семин. по вект. и тенз. анал., 1961, XI, 293-308.

13. Муллари Р. Р. // Уч. зап. Тартуск. ун-та, 1962, 129, 62-73.

14. Евтушик $\mathcal{I}$. E. и др. Дифференциально-геометрические структуры на многообразиях. Пробл. геом. Т. 9. М., ВИНИТИ АН СССР, 1979.

Tartu State University

Received

Jan. 27,1989

Proc. Estonian Acad. Sci. Phys. Math., 1989, 38, N 4, 457-459

удК 537.226 .4

P. KONSIN and T. ORD

\section{CHANGE OF THE ORDER OF FERROELECTRIC PHASE TRANSITION UNDER HYDROSTATIC PRESSURE}

\author{
P. KONSIN, $T$, ORD, SENJETTELEKTRILISE FAASISIIRDE LIIGI MUUTUMINE HODROSTAA- \\ TILISE ROHU TOIMEL \\ П. КОНСИН, Т. ЭРД. ИЗМЕНЕНИЕ РОДА СЕГНЕТОЭЛЕКТРИЧЕСКОГО ФАЗОВОГО ПЕРЕ- \\ ХОДА С ГИДРОСТАТИЧЕСКИМ ДАВЛЕНИЕМ
}

\section{(Presented by V. Hizhnyakov)}

In this paper, the possibilities of changing the order of ferroelectric phase transition under high hydrostatic pressure have been investigated on the phenomenological level. Such effect or a corresponding tendency has been found, e.g., in the monocrystals $\mathrm{BaTiO}_{3}\left[{ }^{1-3}\right]$, SbSI $\left.{ }^{4-7}\right]$, KDP $\left[{ }^{8-11}\right], \mathrm{Sn}_{2} \mathrm{P}_{2} \mathrm{~S}_{6}\left[{ }^{12}\right]$, TGSe [13-15].

Let us start from the expansion of the free energy in powers of the order parameter $y$ and the strain $\varepsilon$ for a liquid-like model of a ferroelectric in which, besides the standard terms, the terms with the coefficients $h, f$ and $w$ have been taken into account:

$$
F=\alpha(T) y^{2}+\beta y^{4}+\gamma y^{6}+\frac{1}{2} c \varepsilon^{2}+g \varepsilon y^{2}+\frac{1}{2} h \varepsilon^{2} y^{2}+f \varepsilon y^{4}+\frac{1}{3} w \varepsilon^{3} .
$$

Here $c$ and $g$ are the elastic and electrostrictive constants. 